\title{
Revisión de los ensayos clínicos sobre prevención del daño neurológico en el traumatismo craneoencefálico grave y análisis de su fracaso terapéutico
}

\author{
G.R. Boto; P.A. Gómez; J. De la Cruz* y R.D. Lobato
}

Servicio de Neurocirugía y Unidad de Epidemiología Clínica*. Hospital 12 de Octubre. Madrid.

\section{Resumen}

En la pasada "Década del Cerebro" se han sometido a ensayo clínico distintos fármacos neuroprotectores frente al traumatismo craneoencefálico grave (TCEG) sin que ninguno de ellos haya mejorado de manera significativa el pronóstico final de estos pacientes. La eficacia neuroprotectora de estas sustancias, confirmada en los modelos animales, no ha podido trasladarse al ámbito clínico, creando gran decepción en la comunidad científica.

Este trabajo se ha estructurado en tres partes: en la primera, se resumen los mecanismos fisiopatológicos involucrados en el TCEG, diana de los fármacos neuroprotectores. En la segunda parte se revisan cuáles han sido los ensayos clínicos más importantes sobre TCEG llevados a cabo en estos últimos años y en la tercera, se analizan los motivos que pueden explicar el "fracaso" terapéutico de estos fármacos.

PALABRAS CLAVE: Ensayos clínicos. Traumatismo craneoencefálico grave. Neuroprotección.

Overview of the recent clinical trials in severe head injury and analysis of their therapeutic failure

\section{Summary}

During the past "Decade of the Brain" several neuroprotective agents have been tested in phase III clinical trials for severe head injury (SHI) but unfortunately none of them significantly improved the outcome of these patients. In contrast to the success achieved by these drugs in animal laboratory studies, the results in terms of neuroprotection in the clinical setting have been disappointing.

This paper has been divided in three parts: in the first one, we summarize the pathophysiological mechanisms related to SHI, targeted by the neuroprotective

Recibido: 08-01-04. Aceptado: 12-11-04 agents. In the second part we review the main clinical trials carried out for SHI to date, and in the third one, we analyze the possible reasons that explain why these agents have failed to show efficacy.

KEY WORDS: Clinical trials. Severe head injury. Neuroprotection.

1. Mecanismos fisiopatológicos involucrados en el traumatismo craneoencefálico grave (TCEG), diana de los ensayos clínicos

De manera general, en la fisiopatología del TCEG se distinguen tres tipos de "daño" o "lesión". Se denomina daño cerebral "primario" a aquél que se genera en el mismo momento que un sujeto de unas determinadas características recibe un tipo particular de traumatismo. Sin embargo, tan importante como el daño primario es el "secundario", generalmente de carácter isquémico y causado por diversos factores como la hipotensión, la hipoxia, la aparición de masas intracraneales, el "swelling" o la elevación de la presión intracraneal (PIC). A diferencia del primario, el daño secundario puede evitarse, al menos en parte. En el llamado daño cerebral "terciario" se resumen todos los fenómenos bioquímicos que se desencadenan tras el traumatismo. El manejo actual del TCEG se basa en la prevención del daño primario, con medidas como las nuevas reglamentaciones del tráfico rodado, y en el control de las lesiones secundarias y terciarias. Recientemente se ha podido demostrar que el TCEG es un proceso dinámico, que evoluciona en horas o incluso días. Es precisamente esta naturaleza dinámica, la que permite aplicar nuevas terapias que tratan de minimizar el daño progresivo o que promueven precozmente los procesos encaminados a la reparación celular $^{34,52,53}$.

Aunque la fisiopatología del TCEG es compleja por la concurrencia de múltiples mecanismos actuando a la vez o secuencialmente, posiblemente todos ellos jueguen un papel más o menos relevante y su común denominador final sea el mismo: pérdida de la homeostasis iónica y daño celular secundario a la entrada de calcio al interior celular. 
Por tanto, la probabilidad de demostrar eficacia cuando un fármaco se dirija hacia varios de estos mecanismos simultáneamente sea mayor que cuando el tratamiento tenga como objeto un único mecanismo. Otros, por contra, creen que cuando se desarrollan ensayos clínicos, ha de identificarse el mecanismo fisiopatológico concreto sobre el que se quiere actuar, cuyo efecto independiente y significativo a la hora de empeorar el pronóstico debe ser bien conocido, para posteriormente encontrar una terapia específica frente a dicho mecanismo, en una muestra adecuada de sujetos y con una medida pronóstica concreta ${ }^{8,35,52}$.

Los mecanismos fisiopatológicos del TCEG, principales "objetivos" de los distintos ensayos clínicos se describen someramente a continuación ${ }^{8,52}$ :

\subsection{Daño isquémico}

Aproximadamente el $90 \%$ de los pacientes que fallecen tras un TCEG muestran signos de isquemia cerebral en los exámenes histopatológicos. Alrededor de un tercio de los pacientes con TCEG soportan un período muy precoz (primeras 6 horas tras el traumatismo) de importante disminución del flujo sanguíneo cerebral (FSC), proceso isquémico que origina entrada de calcio al interior de las células, inactivando enzimas y mecanismos celulares protectores, así como inicio del metabolismo anaerobio, generación de lactato e hinchazón celular ("edema citotóxico"). Todos estos procesos conducen a elevación de la PIC, la cual, a su vez, hace disminuir nuevamente el FSC, cerrando de esta manera el círculo vicioso. Por otro lado, las quininas, formadas en el tejido cerebral dañado, también se han visto involucradas en la alteración de la microcirculación, el desarrollo de hinchazón celular y la aparición de isquemia. De hecho, se ha demostrado en experimentación animal que su inhibición (antagonistas de la bradiquinina), reduce el edema cerebral traumático.

\subsection{Lesión axonal difusa (LAD)}

Alrededor del 25\% de los pacientes que fallecen tras un TCEG presentan hallazgos histopatológicos compatibles con LAD. Sin embargo, estudios actuales empleando análisis inmunohistoquímicos con $\beta$-amiloide demuestran que la LAD puede estar presente en más del $90 \%$ de los pacientes con TCEG. Recientemente, se ha descubierto que existe una secuencia de cambios progresivos en los axones dañados tras un TCEG, abriendo de esta forma la posibilidad de revertir un proceso que se creía completado en milisegundos tras el impacto y, por tanto, irreversible. Por esta razón, se están desarrollando terapias, como la ciclosporina, específicamente dirigidas contra esta lesión axonal "progresiva".

\subsection{Contusiones y hematomas cerebrales}

Es conocido que el FSC puede caer por debajo de los niveles de isquemia en las regiones periféricas a las contusiones y hematomas postraumáticos. A su vez, estas zonas experimentan aumento de las demandas metabólicas $\mathrm{y}$, consecuentemente, mayor utilización de glucosa, que, de no ser adecuadamente corregidas provocarán hinchazón de los astrocitos perilesionales. Nuevamente, este fenómeno acabará originando elevación de la PIC y ésta, nueva reducción en el FSC, cerrando así el círculo vicioso. Diversos estudios en animales y humanos han demostrado que existe una mayor liberación de glutamato en el tejido cerebral que rodea estas lesiones focales, el cual produce despolarización de las membranas celulares, entrada de sodio y calcio al interior celular con salida de potasio, "swelling" astrocitario, aumento de PIC, y consecuentemente mayor reducción de FSC y nuevamente mayor liberación de glutamato ${ }^{46}$.

\section{Resumen de los ensayos clínicos recientes en el TCEG}

El ensayo clínico se define como aquel ejercicio científico que trata de averiguar la eficacia de agentes, sistemas, regímenes o procedimientos, desde un punto de vista preventivo, diagnóstico o terapéutico. Podemos diferenciar cuatro fases en los ensayos clínicos ${ }^{20}$ :

Fase I: determinan el mecanismo de acción, la seguridad del fármaco y sus márgenes de dosificación. Se realizan por la industria farmacéutica en voluntarios sanos.

Fase II: proporcionan estimación inicial de la eficacia y ajuste de la dosis a emplear. Se utilizan para determinar si merece la pena someter al fármaco en cuestión a la fase III. Precisan diseño estadístico y un tamaño muestral habitualmente menor a 100.

Fase III: tratan de demostrar que el nuevo fármaco es mejor que el empleado hasta entonces o que el placebo. También determinan seguridad y dosificación. Es la última fase en el proceso de aprobación de los fármacos por las autoridades sanitarias.

Fase IV: son estudios "postmarketing" realizados tras la aceptación del fármaco.

En la pasada "Década del Cerebro" se han evaluado mediante ensayos clínicos fase III frente al TCEG, tanto procedimientos terapéuticos como fármacos neuroprotectores que actúan sobre los mecanismos fisiopatológicos que hemos descrito en un trabajo anterior (Neurocirugía 2004. 15: 233-247), sin que ninguno de ellos haya conseguido demostrar eficacia. Entre los primeros cabe citar la hiperventilación ${ }^{50}$, el manitol ${ }^{49}$, los barbitúricos ${ }^{48}$, y la hipotermia ${ }^{25,28}$, pero nosotros centraremos nuestra revisión en los siguientes cuatro tipos de fármacos neuroprotectores. 
(Tabla 1) 2,8,35,52:

\section{1. Corticoides}

En un ensayo clínico con 396 pacientes que sufrieron TCEG, se investigó el beneficio de la triamcinolona frente a placebo en las primeras 4 horas tras el traumatismo. Aunque el grupo de la triamcinolona registró una mortalidad menor y un pronóstico mejor, la diferencia no fue significativa. En un subgrupo de pacientes con lesión focal, dentro de este mismo ensayo clínico, se demostró un efecto estadísticamente significativo de la triamcinolona, pero sin suficiente firmeza como para tomar decisiones clínicas basadas en este hallazgo ${ }^{26}$. Por tanto, no existe clara indicación para el uso de estos fármacos en el traumatismo craneoencefálico (TCE) en general, ni en el TCEG en particular ${ }^{1}$. Sin embargo, un metaanálisis de los resultados de todos los ensayos clínicos relacionados con el uso de los corticoides, ha mostrado que estos agentes podrían reducir la mortalidad del TCE de cualquier grado en un $2 \%$, aunque para confirmar definitivamente este dato sería necesario realizar un ensayo clínico con unos 20.000 pacientes. Dicho estudio, que se conoce como "CRASH trial" ("corticosteroids after significant head injury"), se inició en Marzo del 2001 y es en la actualidad el ensayo clínico sobre TCE que más sujetos ha conseguido incluir (en poco más de un año ya tenía 3.000 pacientes si bien sólo un $40 \%$ de ellos eran TCEGs) ${ }^{43}$.

\subsection{Antagonistas del calcio}

El nimodipino previene y reduce el daño isquémico en pacientes con hemorragia subaracnoidea espontánea y su eficacia ha sido estudiada en varios ensayos clínicos relacionados con el TCE. En el primero de ellos ("Head Injury Trial" (HIT) I) ${ }^{5}$, del grupo cooperativo británico-finlandés, se estudiaron 351 pacientes con TCEG, encontrando un $8 \%$ de mejoría a los seis meses en aquéllos que habían recibido nimodipino frente al grupo placebo, pero la diferencia no alcanzó significación estadística. En el HIT $\mathrm{II}^{4}$, del grupo europeo, se estudiaron 852 pacientes con TCEG pero el nimodipino tampoco demostró beneficio significativo; sin embargo, en un subgrupo de pacientes con hemorragia subaracnoidea traumática (HSAt) dentro de este mismo ensayo, la mortalidad y el pronóstico desfavorable fueron significativamente menores en el grupo que recibió nimodipino. En el HIT III ${ }^{27}$ se estudiaron 123 pacientes con TCE y evidencia de HSAt en la tomografía computarizada (TC) craneal, encontrando una reducción significativa en el pronóstico desfavorable del $46 \%$ al $25 \%$ en el grupo tratado con nimodipino; no obstante, el $25 \%$ de los pacientes de este estudio tenían un TCE leve y no pudo confirmarse la presencia de hemorragia subaracnoidea en el $21 \%$ de los casos, cuando las TCs craneales fueron revisados por un comité de expertos, por lo que el uso de este fármaco en la HSAt no ha llegado a generalizarse ${ }^{33}$. Un análisis combinado de los datos del HIT I, II y III, recogió un total de 460 pacientes con HSAt demostrando beneficio significativo en el uso del nimodipino (aumento del pronóstico favorable -categorías de buena recuperación (BR) e incapacidad moderada (IM) en la "Glasgow Outcome Scale" (GOS)de un $45 \%$ a un $56 \%$ ). Sin embargo, este estudio no está exento de errores metodológicos desde el punto de vista estadístico (poblaciones y criterios de inclusión diferentes en cada uno de los ensayos, e inclusión del subgrupo que ha servido para generar la hipótesis en el análisis final) y además, sus resultados no han podido confirmarse en estudios similares. El HIT IV tampoco ha demostrado efecto beneficioso del nimodipino en la HSAt, aunque sus resultados no han sido aún publicados.

\subsection{Secuestradores de radicales libres}

El tirilazad es un 21-aminoesteroide que inhibe la peroxidación lipídica, uno de los mecanismos que forman parte del daño terciario en el TCEG. Este fármaco ha sido estudiado en dos ensayos clínicos de pacientes con TCEG o traumatismo craneoencefálico moderado (TCEM). El estudio norteamericano se suspendió, tras incluir un total de 1.155 sujetos, después de encontrar una mortalidad significativamente mayor en el grupo que recibió tirilazad. En el estudio europeo-australiano ${ }^{38}$, la eficacia de tirilazad se evaluó en 1.120 pacientes, sin hallar efecto significativo; en el subgrupo de pacientes varones con HSAt, dentro de este mismo ensayo clínico, el tirilazad demostró reducción de la mortalidad frente al placebo, con significación estadística. Sin embargo, ni este estudio ni el norteamericano estaban bien balanceados con respecto a determinadas variables pronósticas (frecuencia de hipotensión e hipoxia previas al tratamiento, e incidencia de hematoma epidural), favoreciendo al grupo placebo.

Otro secuestrador de radicales libres ("polyethylene glycol-conjugated bovine superoxide dismutase" (PEGSOD) -pegorgotein-) fue evaluado en 463 pacientes con TCEG, mediante ensayo clínico. Se encontró un incremento del $9 \%$ de pronóstico favorable en el grupo tratado con este fármaco respecto al grupo placebo, pero sin alcanzar tampoco significación estadística ${ }^{54}$.

\subsection{Antagonistas de los receptores de glutamato/N-metil- D-aspartato (NMDA)}

Diversos aminoácidos excitadores, principalmente glutamato, se liberan al espacio extracelular cerebral tras un TCEG, de tal manera que a mayor gravedad de éste, niveles más elevados de todos ellos en el líquido cefalorraquídeo y 


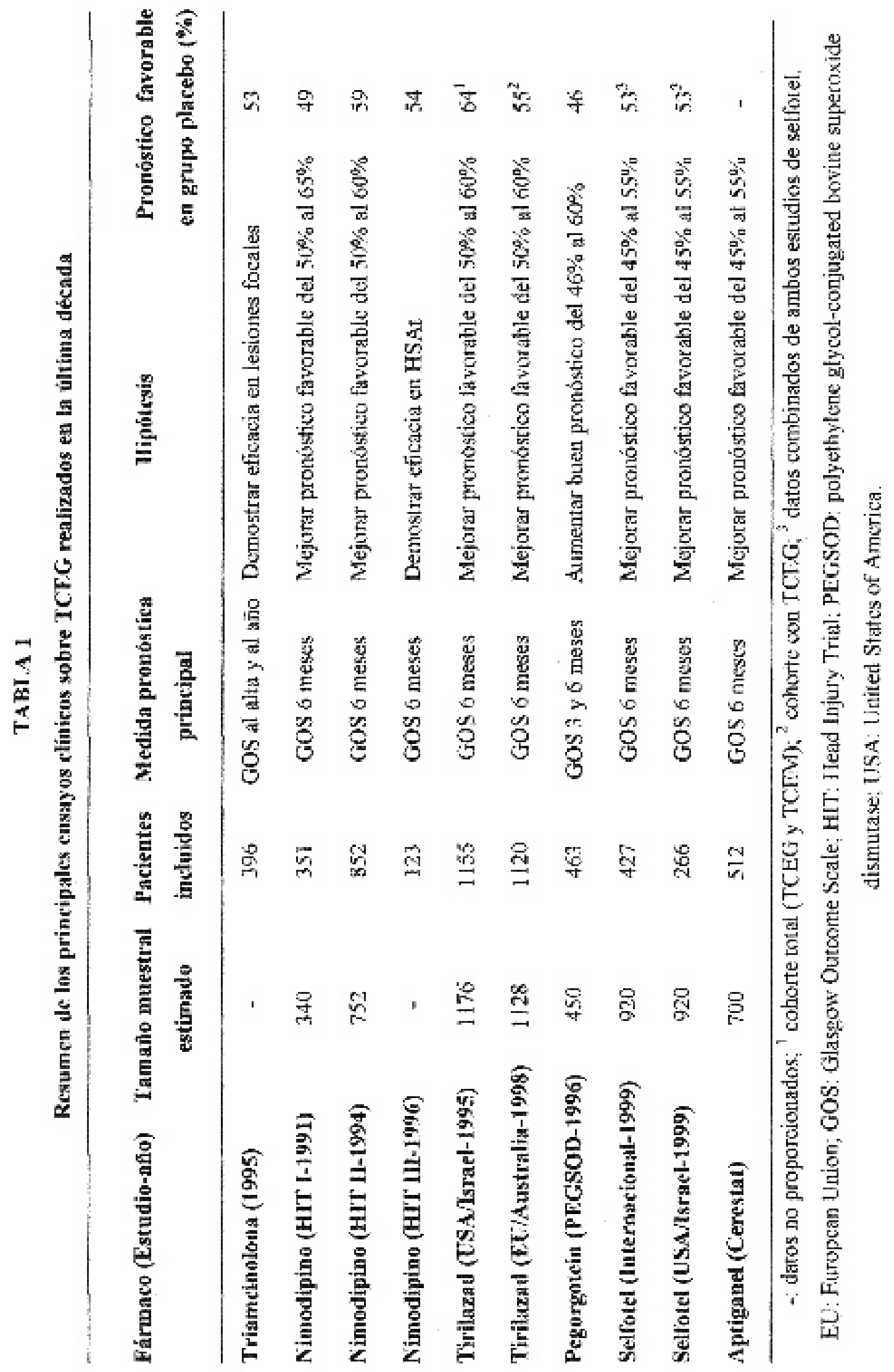


peor pronóstico ${ }^{7,32,46,55}$. A partir de diversos estudios experimentales se sabe que los antagonistas de los receptores de NMDA pueden impedir el daño originado por estos aminoácidos. Con este fin, se evaluó la utilidad del selfotel, un antagonista NMDA competitivo, mediante dos ensayos clínicos que trataron de recoger un total de 920 pacientes con TCEG en cada estudio, para detectar una diferencia en el pronóstico favorable del 10\%. Ambos fueron suspendidos, tras comprobar que en otros dos ensayos clínicos que se estaban realizando, paralelamente con este mismo fármaco frente al ictus cerebral, había más muertes y sucesos adversos en el grupo de tratamiento. Además, aunque los ensayos clínicos se hubieran completado, probablemente no hubiera sido posible demostrar beneficio, ya que tras analizar un total de 693 pacientes entre ambos estudios, el pronóstico favorable fue mayor en el grupo placebo que en el del selfotel ${ }^{41}$.

En 1996 se inició un ensayo clínico con aptiganel (Cerestat), un antagonista NMDA no competitivo, que intentó recoger un total de 700 pacientes con TCEG, para detectar un incremento en el pronóstico favorable del 10\%. El estudio se suspendió en 1997 tras analizar los primeros 340 sujetos y encontrar una diferencia, según la GOS a los tres meses, de sólo $1,1 \%$ a favor del fármaco.

\section{Análisis del "fracaso" de los ensayos clínicos en el TCEG}

Son requisitos imprescindibles para realizar un ensayo clínico que el estudio sea prospectivo, se realice en él algún tipo de intervención, esté correctamente diseñado, sus datos se analicen adecuadamente y sea capaz de superar un control de calidad. Debería llevarse a cabo únicamente en aquellos centros donde la inclusión de pacientes, el seguimiento de los mismos y el manejo de los datos fuera escrupuloso. Aquellos sujetos cuyo seguimiento no esté completamente asegurado (extranjeros, sujetos sin hogar,...), no deberían incluirse en los ensayos clínicos ya que su pérdida puede sesgar el estudio y resulta económicamente muy costosa $a^{11,20,21}$

No todos los fármacos potencialmente beneficiosos en el TCEG pueden someterse a ensayo clínico, habida cuenta del elevado coste de los mismos, principalmente cuando son multicéntricos ${ }^{40}$. Aunque en la mayoría de los ensayos clínicos realizados hasta ahora el fármaco parece superior al placebo, la diferencia no alcanza significación estadística. Sustancias como los antagonistas del calcio, los lazaroides (21-aminoesteroides), y los antagonistas competitivos y no competitivos de los receptores de glutamato, han demostrado eficacia neuroprotectora en modelos experimentales pero estos resultados no han podido corroborarse en el campo clínico, creando gran decepción en la comunidad científica ${ }^{9}$. Quizás algunos de ellos no sean realmente útiles en el TCEG. No obstante, características específicas de las poblaciones estudiadas, o problemas en el propio diseño de los ensayos clínicos, podrían haber contribuido a estos resultados desalentadores, que a continuación pasamos a analizar $2,6.6,15,20,22.23,29,35,36,44,45$.

\subsection{Aspectos farmacológicos}

Para que un fármaco muestre efectividad en un ensayo clínico deben cumplirse los siguientes requisitos esenciales: a) su eficacia ha sido demostrada previamente en modelos animales, b) su seguridad y tolerabilidad se han constatado en pacientes con TCE; c) el mecanismo lesional sobre el que tiene efecto ocurre en la población en la que se está realizando el estudio; d) alcanza niveles plasmáticos terapéuticos y adecuada concentración en el tejido cerebral, incluso en períodos de FSC disminuido; e) es activo en el preciso momento que se produce el mecanismo lesional; y f) la escala pronóstica empleada para detectar su efecto beneficioso posee suficiente sensibilidad. A la hora de someter un fármaco a la fase III de un ensayo clínico, en la que se trata de demostrar que aquél es mejor que el placebo, todas sus características farmacológicas deberían haber sido completamente clarificadas en las dos fases previas. De esta forma, los estudios en fase III quedarían "hechos a medida" para una población específica de estudio.

Uno de los problemas de los modelos animales estriba en que, habitualmente, se centran en un aspecto fisiopatológico concreto, olvidando que en el TCE concurren múltiples mecanismos lesionales que interaccionan entre ellos. Por tanto, serían necesarios varios modelos para aproximarse a la realidad clínica del paciente. Pese a esto, y en aras de la sencillez, se diseñan ensayos clínicos con fármacos dirigidos a mecanismos fisiopatológicos concretos o, a lo más, a un número limitado, como el nimodipino, de tal manera que sea posible reducir el número de sujetos necesario para demostrar un efecto beneficioso y por ende, el de los expuestos a efectos indeseables. Otra cuestión digna de mención es que la mayoría de los estudios con animales se han ocupado más del TCEM que del TCEG, y no han tenido en cuenta cuestiones tan importantes como los insultos secundarios (hipotensión e hipoxia) o la presencia de lesiones extracraneales asociadas, tan frecuentes en el TCEG de los humanos, amén de que isquemia y LAD sean probablemente las características fundamentales que diferencian el TCEG del TCEM.

Varios ensayos clínicos sobre TCEG se desarrollaron a partir de una mínima evidencia de eficacia en los modelos animales, sin llegar a demostrar que el mecanismo contra el que actuaba el fármaco, ocurriera realmente en el humano. Tal es el caso del tirilazad y del PEGSOD, que se diseñaron sin verificar que los radicales libres se liberaran en el cerebro traumatizado del hombre. Circunstancia parecida 
sucedió con los aminoácidos excitadores, principalmente glutamato, cuya neurotoxicidad ocurre fundamentalmente en pacientes con contusiones focales, hematomas subdurales agudos y fenómenos isquémicos secundarios, y rara vez en pacientes sin daño estructural. Sin embargo, el $40 \%$ de los pacientes en los ensayos clínicos del selfotel no presentó ninguna lesión focal. De igual modo, la isquemia difusa es más frecuente que la focal en el TCEG y, sin embargo, fármacos como los mismos antagonistas de los receptores de glutamato-NMDA, sólo tienen utilidad en la isquemia focal. Finalmente, pese a que posiblemente la LAD sea el mecanismo fisiopatológico que más incapacidad causa entre los supervivientes del TCEG, los trabajos sobre esta entidad se han centrado hasta la fecha más en el ámbito experimental que en el clínico.

En diversos ensayos clínicos no se ha demostrado beneficio del fármaco por la inadecuada penetración del mismo en el cerebro, de tal manera que, por ejemplo, sólo tras la realización de los ensayos del tirilazad y del PEGSOD se supo que las concentraciones mayores de estos fármacos se alcanzan en la microcirculación cerebral y que escasamente penetran en el cerebro dañado. Este hecho explicaría el beneficio que mostró el tirilazad en un subgrupo de pacientes varones con HSAt. Además, los niveles plasmáticos de tirilazad fueron menores en mujeres que en hombres, incluso a veces por debajo del umbral terapéutico y su aclaramiento, aproximadamente un 40\%, mayor en las primeras que en los segundos. Por tanto, la dosificación en las mujeres podría haber sido insuficiente ${ }^{39}$. Otra cuestión en la que no se reparó es que el metabolismo del tirilazad aumenta en aquellos pacientes que reciben fenitoína, fármaco con frecuencia empleado como profilaxis de las crisis convulsivas en el TCEG.

Los estudios con animales han hecho hincapié en la importancia de una dosificación correcta del fármaco. En ellos, se administra la dosis por peso corporal, a diferencia de muchos ensayos clínicos en humanos en los que la dosificación es la misma para todos los pacientes. Aun así, en ambos casos se podría incurrir en infradosificación, especialmente en fármacos cuyo mecanismo de acción es el antagonismo competitivo, de tal forma que hoy se sabe, por ejemplo, que las concentraciones cerebrales de glutamato en pacientes con TCEG llegan a los 100 micromol/L y las de selfotel, en condiciones normales, son de aproximadamente 2 micromol/L (e incluso menores en el cerebro traumatizado, debido a la reducción del flujo sanguíneo local), cifras claramente insuficientes para un antagonista competitivo del receptor de NMDA como éste. En este contexto, es especialmente útil la "microdiálisis cerebral", que permite, de forma individualizada, medir la concentración del fármaco en el cerebro humano traumatizado, evaluar su efecto para calcular así la dosis apropiada (la llamada "dosis según el efecto", en la que, por ejemplo, se mide la concentración de glutamato cerebral tras recibir el fármaco y después se ajusta la dosis de éste), determinar la denominada "ventana terapéutica" y obtener substratos del metabolismo cerebral para inferir posibles mecanismos patológicos.

La determinación del momento apropiado para realizar la intervención terapéutica, o "ventana terapéutica", es de crucial importancia para que el fármaco pueda demostrar beneficio en los ensayos clínicos, de tal manera que la administración del mismo prematuramente, excesivamente tarde o durante un período de tiempo inadecuado, podría invalidar el estudio. Además, para un mismo mecanismo fisiopatológico las ventanas terapéuticas en animales pueden diferir de las de los humanos y aunque se cree que son mayores en estos últimos, no existe absoluta evidencia. Los estudios en animales han demostrado que la medicación debe administrarse lo antes posible tras el traumatismo, ya que generalmente, después de la primera hora desaparece el efecto de la misma. Sin embargo, en los principales ensayos clínicos se ha utilizado habitualmente una ventana de 8 horas (PEGSOD, selfotel, Cerestat), oscilando ocasionalmente entre las 4 (corticoides, tirilazad) y las 12 horas (nimodipino). Una de las razones que obliga a ventanas tan amplias radica en la obligatoriedad de obtener el consentimiento informado de los familiares del paciente. Por este motivo, lo ideal sería utilizar medicaciones que, careciendo de efectos secundarios, como el PEGSOD, pudieran administrarse en el mismo lugar del accidente, obviando así la necesidad de dicho consentimiento. De hecho, la nueva legislación española sobre ensayos clínicos, recientemente aprobada, soslaya la obligatoriedad del consentimiento informado bajo ciertas condiciones.

\subsection{Heterogeneidad de los pacientes}

Pese a criterios estrictos de inclusión y exclusión, los pacientes, que han formado parte de cada uno de los ensayos clínicos sobre TCEG, presentan diferencias en su estado clínico y en el tipo de lesiones que portan, careciendo por tanto de homogeneidad. Para amortiguar este fenómeno se utiliza la "randomización", que consiste en distribuir estos aspectos por igual entre ambos grupos de tratamiento (fármaco y placebo). Incluso con tamaños muestrales en torno a 800 ó 1.000 pacientes, considerados más que suficientes para un ensayo clínico en el TCEG, pueden aparecer importantes desequilibrios entre ambos grupos, como ocurrió en los ensayos del tirilazad. Estos desequilibrios pueden reducirse "estratificando" a los pacientes por determinadas variables, es decir, ajustando por sus indicadores pronósticos, principalmente edad, puntuación en la "Glasgow Coma Scale" (GCS), reactividad pupilar, presencia de hipotensión y/o hipoxia y hallazgos en la TC craneal. Por tanto, resultaría útil dirigir los ensayos clínicos a pacientes 
homogéneos, es decir, con un estado clínico y unas lesiones en la TC craneal, similares. Tanto la estratificación previa a la randomización como el uso de aquélla para corregir desequilibrios entre grupos randomizados, mediante el análisis estadístico multivariable, mitigan los problemas de heterogeneidad, reducen la influencia pronóstica inicial y mejoran el poder estadístico del estudio, aspecto éste particularmente útil dado que, por razones económicas, el número de pacientes que uno puede habitualmente contemplar en los ensayos clínicos es limitado.

\subsection{Tamaño muestral}

El objetivo principal en la mayor parte de los ensayos clínicos sobre TCEG ha sido detectar de un $10 \%$ a un $12 \%$ de mejoría en el pronóstico favorable (categorías de BR e IM en la GOS) a los seis meses del traumatismo. Para un objetivo de estas características, se estima que el tamaño muestral total requerido es de 800 pacientes (dos grupos de 400 sujetos), para un poder estadístico (suponer que el tratamiento es eficaz cuando lo es) del $80 \%$ y un nivel de significación del 0,05 . Si el fármaco fuera efectivo sólo en determinados tipos de traumatismos, encontrar una mejoría global del 10\% resultaría todavía más dificil. Diversos estudios de revisión han confirmado que hasta finales de 1998, se habían publicado unos 208 ensayos clínicos randomizados y controlados sobre el tratamiento o la rehabilitación de pacientes con TCE. Ninguno tenía suficiente tamaño muestral para detectar fiablemente una reducción en el riesgo de muerte o incapacidad del 5\% y sólo un $4 \%$ eran suficientemente grandes para detectar una reducción del $10 \%$, asumiendo un error alfa (suponer que el tratamiento es eficaz cuando no lo es) del $5 \%$, un poder del $80 \%$ (equivalente a un $20 \%$ de error beta -suponer que el tratamiento no es eficaz cuando lo es-) y una prevalencia en el grupo control del $20 \%$. Si la prevalencia hubiera sido mayor, la dificultad para detectar una reducción en el riesgo de muerte o incapacidad para esos tamaños muestrales, también habría aumentado. Por tanto, la gran mayoría de los ensayos clínicos realizados hasta ahora han carecido de un tamaño muestral adecuado y probablemente no hayan estado bien diseñados. Bajo esta tesitura, ha aparecido la corriente de los llamados " lumpers" o defensores de los megaensayos clínicos ${ }^{22}$.

Dado que el TCEG es una entidad muy frecuente, si se encontrara un tratamiento que redujera la morbi-mortalidad, aunque fuera en un porcentaje pequeño, el número de pacientes que podría obtener beneficio sería muy elevado. Para detectar estos efectos moderados del tratamiento, es necesario evitar los errores aleatorios, generalmente con muestras suficientemente grandes, y los sistemáticos o sesgos, mediante un diseño adecuado. Por tanto, aumentar sustancialmente el número de pacientes estudiados tiene por objeto incrementar el poder necesario para detectar diferencias que pasarían desapercibidas con muestras más pequeñas, pero dicha estrategia tiene una clara limitación económica. Una de las maneras de soslayar esta dificultad consiste en restringir la recogida de datos a las variables esenciales $^{30}$.

El problema es de tal envergadura que aun sumando todos los participantes de los ensayos clínicos de un mismo fármaco o terapia realizados hasta ahora en el TCEG, no se alcanzaría un tamaño muestral suficiente. De hecho, el número total de participantes randomizados en todos los ensayos clínicos disponibles sobre TCE (16.613 sujetos) es notablemente inferior al número de sujetos involucrados en algunos ensayos clínicos sobre cardiopatía isquémica o ictus cerebral. Además, las asignaciones económicas de los diversos organismos públicos y privados para ensayos clínicos sobre TCEG son menores que las destinadas a los del cáncer o a los de las enfermedades cardiovasculares.

\subsection{Población diana}

En los ensayos clínicos realizados hasta el momento se han incluido pacientes con pocas probabilidades de sobrevivir a priori, junto con otros que muy posiblemente alcancen un pronóstico favorable. En ambos subgrupos es difícil que un fármaco demuestre beneficio clínico relevante. Por ello, parece fundamental optimizar el tipo y número de pacientes que se incluyan en futuros estudios, seleccionando subgrupos de individuos con un pronóstico "intermedio", es decir, aquéllos que posiblemente sobrevivan con incapacidad (por ejemplo, entre el $20 \%$ y el $80 \%$ de probabilidad de un pronóstico favorable), y descartando los que alberguen grandes posibilidades de fallecer o por contra, de tener un pronóstico favorable (pacientes con probabilidad de un pronóstico favorable menor al $20 \%$ o mayor al $80 \%$, respectivamente). De esta manera, para el mismo poder estadístico, sería posible reducir el tamaño muestral hasta en un 30\%. Un paso siguiente consistiría en ajustar por diversos indicadores pronósticos, de tal manera que, además de reducir el tamaño muestral, aumentara el poder estadístico al evitar la heterogeneidad. Por tanto, los ensayos clínicos deberían dirigirse hacia una población de riesgo "intermedio", si se pretende encontrar un beneficio del $10 \%$, o bien buscar un efecto más modesto, entre el $5 \%$ y el $8 \%$, si se va a manejar la población global. Esta política de ensayos clínicos "dirigidos", es la defendida por los llamados "dividers", contrarios a los megaensayos de los que son partidarios los "lumpers" $30,31$.

Una potencial desventaja de este esquema de trabajo estriba en que los resultados del ensayo sólo serían aplicables a una subpoblación concreta de pacientes, limitando así el uso de la medicación, con el consiguiente desagrado de las compañías farmacéuticas. Sin embargo, parece 
lógico tratar primero de demostrar eficacia en los pacientes con mayor potencial de beneficio y después ampliar las indicaciones al resto, ayudados de nuevos estudios.

\subsection{Dicotomización de la GOS}

El objetivo final en todos los ensayos clínicos recientes sobre TCEG ha sido determinar el pronóstico, al menos a los seis meses del traumatismo, mediante la GOS dicotomizada en favorable (BR/IM) y desfavorable (incapacidad grave (IG)/estado vegetativo (EV)/exitus (EX)). A los tres meses del traumatismo, la distribución del pronóstico tiene forma de "J", dado que la mayoría de los pacientes que fallecen lo hacen precozmente. Entre los seis y los doce meses, esta distribución va cambiando a una forma de "U", ya que los sujetos que no han fallecido experimentan una mejoría progresiva, modificando su clasificación en la GOS, mientras que el número de fallecidos prácticamente no varía. Esta distribución característica del pronóstico en el TCEG, con forma de "U", explica la dicotomía inherente de la GOS, en la que aproximadamente, del $25 \%$ al $40 \%$ de los pacientes mueren y del $30 \%$ al $45 \%$ alcanzan las categorías de BR o IM, con una proporción, por tanto, de pronóstico favorable similar al desfavorable a expensas del bajo porcentaje de pacientes en las categorías de IG o EV. En efecto, entre los pacientes que sobreviven, un alto número alcanza un pronóstico favorable. Resultará difícil, por tanto, conseguir una mejoría en el pronóstico favorable del $10 \%$ a costa de las categorías de supervivientes sin hacer descender la mortalidad, aunque una reducción de ésta, sin mejora en la calidad de vida, tampoco sería aceptable. De hecho, los trabajos publicados indican que cuando los fármacos son efectivos en un subgrupo de pacientes, se reduce la mortalidad y aumenta el pronóstico favorable en dicho subgrupo.

Diversos análisis estadísticos demuestran que incluso cambios sustanciales en la distribución del pronóstico, como la mejoría de éste hasta en un $25 \%$ de pacientes por categoría en tres de las mismas, no conseguirían demostrar un aumento en el pronóstico favorable del 10\%. El efecto de desplazar pacientes entre categorías depende de la distribución pronóstica inicial, de tal manera que será más marcado si la población tiene un pronóstico a priori más desfavorable. De igual forma, será más fácil demostrar eficacia del fármaco evaluado si la distribución del pronóstico en el grupo placebo es más desfavorable. Estos mismos estudios estadísticos han concluido que los análisis dicotómicos (muerto-vivo, desfavorable-favorable) reflejan los efectos del fármaco a estudio sólo si éstos son marcados porque si son sutiles, probablemente se detecten mejor en análisis ordinales $(\mathrm{BR}>\mathrm{IM}>\mathrm{IG}>\mathrm{EV}>\mathrm{EX})$. Por esta razón, un efecto clínicamente relevante, aun estando presente, puede no verse adecuadamente reflejado en la GOS dicotomizada.
La escala pronóstica más utilizada como objetivo final en los ensayos clínicos sobre TCEG es, como ya se ha mencionado, la GOS. Su alta validez y fiabilidad, su capacidad para discriminar entre grupos que son homogéneos desde el punto de vista clínico y social, y su buena correlación con las pruebas neuropsicológicas, han hecho de ella una escala superior a la "Disability Rating Scale" o a los tests neuropsicológicos ${ }^{12}$. Dado que, posiblemente, no todos los pacientes incluidos en la categoría de IG tengan un pronóstico desfavorable y que, por otro lado, determinados pacientes en las categorías de EV o IM debieran incluirse en la de IG, aumentando así el tamaño de este grupo intermedio y aplanando la distribución en "U", algunos autores creen que sería preferible utilizar la "GOS ampliada" (ocho categorías, producto de la subdivisión en dos de las categorías de BR, IM e IG) a la GOS, aunque la fiabilidad interobservador sea menor con aquélla. Por otro lado, los porcentajes de mortalidad aislados resultan especialmente útiles como indicador pronóstico precoz de la eficacia de los fármacos en los ensayos clínicos sobre TCEG, ya que, su determinación es sencilla, la mayor parte de la mortalidad ocurre precozmente tras el traumatismo obviando así la necesidad de seguimientos prolongados (entre el 50\% y el 75\% de los pacientes que fallecen tras un TCEG lo hacen en las primeras 48 ó 72 horas) ${ }^{10,16,18,19,24,51}$, y las cifras de mortalidad permanecen relativamente elevadas tras aquél ${ }^{13,17,53}$.

Se han considerado otros indicadores como objetivo final a medir en los ensayos clínicos sobre TCEG en sustitución de la GOS dicotomizada, aunque está por demostrar si realmente son capaces de reemplazar a las escalas pronósticas convencionales. Entre ellos destacan la PIC, la saturación yugular de oxígeno y más recientemente el llamado "neurodeterioro"3,42. Este último ha sido definido por la ocurrencia de uno o más de los siguientes supuestos: disminución de dos o más puntos en la respuesta motora de la GCS, nueva pérdida de reactividad pupilar, nueva asimetría pupilar mayor o igual a $2 \mathrm{~mm}$, o deterioro neurológico que requiera intervención médica o quirúrgica inmediata. Aquellos pacientes que presentaron uno o más episodios de neurodeterioro tuvieron significativamente mayor morbi-mortalidad (cinco veces más probabilidad de morir y menos de la mitad de probabilidad de tener un pronóstico favorable) que los que no sufrieron ninguno de estos episodios, según pudo extraerse del estudio internacional del selfotel. Posiblemente, una reducción de este neurodeterioro del $10 \%$ sea equiparable a una mejoría significativa en el pronóstico de los pacientes a los seis meses. Si esta fuerte correlación entre neurodeterioro y GOS a los seis meses pudiera verificarse prospectivamente en un ensayo clínico con éxito, no sería necesario esperar un lapso tan dilatado en futuros ensayos para saber si el fármaco es eficaz o no. Sin embargo, factores sistémicos como la fiebre, pueden contribuir a un deterioro neurológico transitorio 
que enmascaren el potencial efecto del fármaco. Además, efectos secundarios de la medicación podrían interpretarse erróneamente como episodios de neurodeterioro. Por tanto, el uso de este indicador está condicionado a la sedación y parálisis del paciente, y ambos, neurodeterioro y mejoría del pronóstico gracias al fármaco, pueden coexistir.

\subsection{Interpretación final del "fracaso"}

A tenor de los buenos resultados obtenidos a la hora de manejar los pacientes con TCEG en las unidades de neurotrauma actuales, como lo demuestra el hecho de que la morbi-mortalidad en los ensayos clínicos fuera menor de lo esperado, no resulta sorprendente que el efecto de los fármacos investigados haya sido modesto. Paradójicamente, a mejor resultado del tratamiento convencional, más difícil será demostrar beneficio de un nuevo fármaco y a la inversa, a peor tratamiento estándar, mayor riesgo de insultos secundarios y más probabilidad de que el nuevo fármaco demuestre eficacia. Para evitar ésto, los centros participantes en los ensayos clínicos deben manejar un protocolo de tratamiento similar.

La ausencia de significación estadística en un ensayo clínico no supone que el fármaco carezca definitivamente de utilidad, sino simplemente que no se ha podido demostrar la hipótesis habitual, es decir una mejoría en el pronóstico favorable de la muestra estudiada del $10 \%$, usando la GOS dicotomizada. Posiblemente algunos de estos fármacos sean beneficiosos aunque no en una medida tan grande como la inicialmente hipotetizada. La decisión de paralizar un ensayo clínico, sin llegar a conocer si realmente el fármaco tiene algún efecto en la población global o en subgrupos específicos, responde en muchas ocasiones más a intereses económicos de las compañías farmacéuticas que a motivaciones científicas ${ }^{14}$ Pese a todo, aunque no haya sido posible demostrar eficacia en ningún ensayo clínico sobre TCEG hasta la fecha, la realización de éstos ha contribuido indudablemente a un mayor conocimiento del neurotrauma en su conjunto.

\section{Conclusiones}

Es sabido que los daños cerebrales "secundario" y "terciario", responsables principales del mal pronóstico tras un TCEG, pueden tratarse farmacológicamente. Sin embargo, ninguna de las sustancias neuroprotectoras dirigidas expresamente frente a estos tipos de daño cerebral y evaluadas mediante ensayo clínico fase III ha conseguido demostrar eficacia hasta la fecha, con las consecuencias que ésto acarrea. Con objeto de llevar a cabo una estrategia uniforme para evaluar nuevos compuestos en futuros ensayos clínicos, obviando posibles errores ahora conocidos y resumidos en este trabajo, se crearon en el pasado dos orga- nizaciones sin ánimo de lucro: la "American Brain Injury Consortium" (ABIC) y la "European Brain Injury Consortium" (EBIC) $)^{37,47}$. El fin primordial de ambas no es otro que coordinar los futuros ensayos clínicos para mejorar el pronóstico final de los pacientes con TCEG.

\section{Agradecimientos}

Este trabajo ha sido financiado en su mayor parte por la beca FIS 99/0554 concedida al Prof Dr. Ramiro Díez Lobato.

\section{Bibliografía}

1. Alderson, P., Roberts, I.: Corticosteroids for acute traumatic brain injury. Cochrane Database Syst Rev 2000; (2): CD000196.

2. Alves, W.M., Eisenberg, H.M.: Head injury trials-past and present. En Narayan RK, Wilberger JE, Povlishock JT (eds). Neurotrauma. New York; McGraw-Hill, 1996; pp. 947967.

3. Ananda, A., Morris, G.F., Juul, N., Marshall, S.B., Marshall, L.F.: The frequency, antecedent events, and causal relationships of neurologic worsening following severe head injury. Executive Committee of the international Selfotel Trial. Acta Neurochir Suppl (Wien) 1999; 73: 99-102.

4. Anónimo: A multicenter trial of the efficacy of nimodipine on outcome after severe head injury. The European Study Group on Nimodipine in Severe Head Injury. J Neurosurg 1994; 80: 797-804.

5. Bailey, I., Bell, A., Gray, J., et al.: A trial of the effect of nimodipine on outcome after head injury. Acta Neurochir (Wien) 1991; 110: 97-105.

6. Bolland, K., Sooriyarachchi, M.R., Whitehead, J.: Sample size review in a head injury trial with ordered categorical responses. Stat Med 1998; 17: 2835-2847.

7. Brown, J.I., Baker, A.J., Konasiewicz, S.J, Moulton, R.J.: Clinical significance of CSF glutamate concentrations following severe traumatic brain injury in humans. J Neurotrauma 1998; 15: 253-263.

8. Bullock, M.R., Lyeth, B.G., Muizelaar, J.P.: Current status of neuroprotection trials for traumatic brain injury: lessons from animal models and clinical studies. Neurosurgery 1999, 45:207-220.

9. Bullock, R.: Experimental drug therapies for head injury. En Narayan, Wilberger, Povlishock, (eds). Neurotrauma. New York; McGraw-Hill, 1996; pp. 375-391.

10. Carlsson, C.A., von Essen, C., Lofgren, J.: Factors affecting the clinical course of patients with severe head injuries. 1. Influence of biological factors. 2. Significance of posttraumatic coma. J Neurosurg 1968; 29: 242-251.

11. Choi, S.C., Bullock, R.: Design and statistical issues in 
multicenter trials of severe head injury. Neurol Res 2001; 23: 190-192.

12. Choi, S.C., Clifion, G.L., Marmarou, A., Miller, E.R.: Misclassifcation and treatment effect on primary outcome measures in clinical trials of severe neurotrauma. J Neurotrauma 2002; 19: 17-22.

13. Choi, S.C., Marmarou, A., Bullock, R., Nichols, J.S., Wei, X., Pitts, L.H.: Primary end points in phase III clinical trials of severe head trauma: DRS versus GOS. The American Brain Injury Consortium Study Group. J Neurotrauma. 1998; 15: 771-776.

14. Choi, S.C., Smith, P.J., Becker, D.P.: Early decision in clinical trials when the treatment differences are small. Experience of a controlled trial in head trauma. Control Clin Trials 1985; 6: 280-288.

15. Clifton, G.L, Choi, S.C., Miller, E.R., et al.: Intercenter variance in clinical trials of head trauma--experience of the National Acute Brain Injury Study: Hypothermia. J Neurosurg 2001; 95: 751-755.

16. Clifton, G.L., Grossman, R.G., Makela, M.E., Miner, M.E., Handel, S., Sadhu, V.: Neurological course and correlated computerized tomography findings after severe closed head injury. J Neurosurg 1980; 52: 611-624.

17. Clifton, G.L., Hayes, R.L., Levin, H.S., Michel, M.E., Choi, S.C.: Outcome measures for clinical trials involving traumatically brain-injured patients: report of a conference. Neurosurgery 1992; 31: 975-978.

18. Clifton, G.L., McCormick, W.F., Grossman, R.G.: Neuropathology of early and late deaths after head injury. Neurosurgery 1981; 8: 309-314.

19. Conroy, C., Kraus, J.F.: Survival after brain injury. Cause of death, length of survival, and prognostic variables in a cohort of brain-injured people. Neuroepidemiology 1988; 7 : 13-22.

20. Contant, C.F. Jr.: Clinical trial design. En Narayan, Wilberger, Povlishock, (eds). Neurotrauma. New York; McGraw-Hill, 1996; pp. 923-945.

21. Corrigan, J.D., Harrison-Felix, C., Bogner, J., Dijkers, M., Terrill, M.S., Whiteneck, G.: Systematic bias in traumatic brain injury outcome studies because of loss to follow-up. Arch Phys Med Rehabil 2003; 84: 153-160.

22. Dickinson, K., Bunn, F., Wentz, R., Edwards, P., Roberts, I.: Size and quality of randomised controlled trials in head injury: review of published studies. BMJ 2000; 320: 1308-1311.

23. Doppenberg, E.M., Bullock, R.: Clinical neuro-protection trials in severe traumatic brain injury: lessons from previous studies. J Neurotrauma 1997; 14: 71-80.

24. Eisenberg, H.M., Levin, H.S.: The desvastated head injury patient. Clin Neurosurg 1988; 34: 572-586.

25. Gadkary, C.S., Alderson, P., Signorini, D.F.: Therapeutic hypothermia for head injury. Cochrane Database Syst Rev 2002; (1): CD001048.
26. Grumme, T., Baethmann, A., Kolodziejczyk, D., et al.: Treatment of patients with severe head injury by triamcinolone: a prospective, controlled multicenter clinical trial of 396 cases. Res Exp Med (Berl) 1995; 195: 217-229.

27. Harders, A., Kakarieka, A., Braakman, R.: Traumatic subarachnoid hemorrhage and its treatment with nimodipine. German tSAH Study Group. J Neurosurg 1996; 85: 82-89.

28. Harris, O.A., Colford, J.M. Jr., Good, M.C., Matz, P.G.: The role of hypothermia in the management of severe brain injury: a meta-analysis. Arch Neurol 2002; 59: 10771083.

29. Hukkelhoven, C.W., Steyerberg, E.W., Farace, E., Habbema, J.D., Marshall, L.F., Maas, A.L.: Regional differences in patient characteristics, case management, and outcomes in traumatic brain injury: experience from the tirilazad trials. J Neurosurg 2002, 97: 549-557.

30. Hukkelhoven, C.W., Steyerberg, E.W., Maas, A.I.: Quality of randomised controlled trials in head injury. Statistical power can be increased. BMJ 2000; 321: 704 (letter).

31. Imberti, R.: Why have recent trials of neuroprotective agents in head injury failed to show. Neurosurgery 2003; 53 : 241-242 (letter).

32. Koura, S.S., Doppenberg, E.M., Marmarou, A., Choi, S., Young, H.F., Bullock, R.: Relationship between excitatory amino acid release and outcome after severe human head injury. Acta Neurochir Suppl (Wien) 1998; 71: 244-246.

33. Langham, J., Goldfrad, C., Teasdale, G., Shaw, D., Rowman, K.: Calcium channel blockers for acute traumatic brain injury. Cochrane Database Syst Rev 2003; (4): CD000565.

34. Levin, H.S., Hamilton, W.J., Grossman, R.G.: Outcome after head injury. En Braakman R (ed). Head Injury. Handbook of Clinical Neurology, Vol 13. Amsterdam; Elsevier Science Publishers B. V., 1990; pp. 367-395.

35. Maas, A.I., Steyerberg, E.W., Murray, G.D., et al.: Why have recent trials of neuroprotective agents in head injury failed to show convincing efficacy? A pragmatic analysis and theoretical considerations. Neurosurgery 1999; 44: 12861298.

36. Machado, S.G., Murray, G.D., Teasdale, G.M.: Evaluation and designs for clinical trials of neuroprotective agents in head injury. J Neurotrauma 1999; 16: 1131-1138.

37. Marmarou, A.: Conduct of head injury trials in the United States: the American Brain Injury Consortium (ABIC). Acta Neurochir Suppl (Wien) 1996; 66: 118-121.

38. Marshall, L.F, Maas, A.I., Marshall, S.B., et al.: A multicenter trial on the efficacy of using tirilazad mesylate in cases of head injury. J Neurosurg 1998; 89: 519-525.

39. Marshall, L.F., Marshall, S.B. : Pitfalls and advances from the international tirilazad trial in moderate and severe head injury. J Neurotrauma 1995; 12: 929-932.

40. Michel, M.E.: Economics of head injury trials. En Narayan, Wilberger, Povlishock, (eds). Neurotrauma. New 
York; McGraw-Hill, 1996; pp. 969-975.

41. Morris, G.F., Bullock, R., Marshall, S.B., Marmarou, A., Maas, A., Marshall, L.F.: Failure of the competitive Nmethyl-D-aspartate antagonist Selfotel (CGS 19755) in the treatment of severe head injury: results of two phase III clinical trials. The Selfotel Investigators. J Neurosurg 1999; 91 : 737-743.

42. Morris, G.F., Juul, N., Marshall, S.B., Benedict, B., Marshall, L.F.: Neurological deterioration as a potential alternative endpoint in human clinical trials of experimental pharmacological agents for treatment of severe traumatic brain injuries. Executive Committee of the International Selfotel Trial. Neurosurgery 1998; 43: 1369-1374.

43. MRC CRASH Trial National Coordinators: Update on progress in the international, multicenter, randomized, controlled trial ofcorticosteroids after significant head injury (Medical Research Council CRASH Trial). Curr Opin Crit Care 2003; 9: 92-97.

44. Murray, G.D.: Use of an international data bank to compare outcome following severe head injury in different centres. Stat Med 1986; 5: 103-112.

45. Narayan, R.K., Michel, M.E., Ansell, B., et al.: Clinical trials in head injury. J Neurotrauma 2002; 19: 503-557.

46. Reinert, M., Khaldi, A., Zauner, A., Doppenberg, E., Choi, S., Bullock, R.: High level of extracellular potassium and its correlates after severe head injury: relationship to high intracranial pressure. J Neurosurg 2000, 93: 800-807.

47. Reinert, M.M., Bullock, R.: Clinical trials in head injury. Neurol Res 1999; 21: 330-338.

48. Roberts, I.: Barbiturates for acute traumatic brain injury. Cochrane Database Syst Rev 2000; (2): CD000033.

49. Roberts, I., Schierhout, G., Alderson, P.: Absence of evi- dence for the effectiveness of five interventions routinely used in the intensive care management of severe head injury: a systematic review. J Neurol Neurosurg Psychiatry 1998; 65: 729-733.

50. Schierhout, G., Roberts, I.: Hyperventilation therapy for acute traumatic brain injury. Cochrane Database Syst Rev 2000; (2): CD000566.

51. Sekulovic, N., Ceramilac, A.: Brain injuries--causes of death and life expectancy. Acta Neurochir Suppl (Wien) 1979; 28: 203-204.

52. Teasdale, G.M., Graham, D.I.: Craniocerebral trauma: protection and retrieval of the neuronal population after injury. Neurosurgery 1998; 43: 723-738.

53. Vollmer, D.G.: Prognosis and outcome of severe head injury. En Cooper PR (ed). Head injury, ed 3. Baltimore; Williams \& Wilkins, 1993; pp. 553-581.

54. Young, B., Runge, J.W., Waxman, K.S., et al.: Effects of pegorgotein on neurologic outcome of patients with severe head injury. A multicenter, randomized controlled trial. JAMA 1996; 276: 538-543.

55. Zhang, X., Qiu, M., Zhang, X., Zhang, J., Zhang, H., Kang, D.: Excitatory amino acids in cerebrospinal fluid and their relations with clinical features and outcomes in acute head injury. Chin Med J (Engl) 1998; 111: 978-981.

Boto, G.R.; Gómez, P.A.; De la Cruz, J.; Lobato, R.D.: Revisión de los ensayos clínicos sobre prevención del daño neurológico en el traumatismo craneoencefálico grave y análisis de su fracaso terapéutico. Neurocirugía 2005; 16 : $39-49$.

Correspondencia postal: Gregorio Rodríguez Boto. Avda. Dr. García Tapia, 159. Escalera D. 4º-B. 28030 Madrid. 